\title{
EFFECTIVE TEMPERATURES OF WHITE DWARFS
}

\author{
J. B. Oke \\ Hale Observatories
}

Of all the fields of stellar astronomy, the study of white dwarfs has been most influenced by work done on proper motions. From proper motion studies, particularly by Luyten and Giclas in recent years, many white dwarf candidates have been found. These candidates have been studied intensively, particularly by Greenstein who has obtained spectra and Eggen who has measured magnitude and colors. As a result, many new white dwarfs have been discovered, and we now have many examples of most types of white dwarfs. It is, therefore, possible to study each group separately and to find relationships between the various types of white dwarfs.

Once the distance of a white dwarf is known, either from its parallax, or from membership in a binary system, then, provided the bolometric correction and effective temperature can be derived, the radius can be determined. From the radius and composition, the mass is derived and stellar evolutionary effects can be studied. Until recently, effective temperatures had to be determined (1) by interpreting UBV colors in terms of black bodies, (2) by interpolation between black boldies and main-sequence stars, or (3) by studying line profiles calculated from rather simple model atmospheres. These methods have yielded approximate temperatures, but the accuracy has been clearly limited.

It became evident several years ago that much better model atmospheres would become available, and that if spectral energy distributions were available, much more accurate effective temperatures and good estimates of the surface gravities could be obtained. The model atmospheres would also provide much better hydrogen-line profiles which would also yield better values of these parameters. Consequently, a program was begun with the 200-inch prime-focus scanner to obtain absolute spectral energy distributions of the white dwarfs brighter than magnitude 13. As new white dwarfs were discovered, a few of the most interesting and easily observed were added to the program. With the completion of the photoelectric multichannel spectrometer two years ago, it became feasible to observe any white dwarf and the program was expanded to include a selection of white dwarfs of each spectral type. At the present time, observations are essentially complete for thirty-two white dwarfs. They include thirteen of type DA, seven of type DB, four of type DF and DG. The remainder are of other types or unusual objects.

Model atmospheres for A-type white dwarfs have been published by Terashita and Matsushima (1969). Kumar is calculating models for cool stars. A student at the California Institute of Technology is making models for helium white dwarfs. Since these last models are not yet available for the present discussion, Planck functions are used to derive temperatures 
of the nonhydrogen-type stars. The observed fluxes are based on the new absolute calibration of $\alpha$ Lyrae recently determined by Oke and Schild (1970). In Figures $1-5$ are shown a few typical examples of observed energy distributions and the fitted model atmosphere fluxes.

The results for stars already analysed can be summarized as follows:

(1) The DA stars have effective temperatures ranging from $13,000^{\circ} \mathrm{K}$ to $50,000^{\circ} \mathrm{K}$.

(2) DB's range from $15,000^{\circ} \mathrm{K}$ to $25,000^{\circ} \mathrm{K}$.

(3) Two stars have $\mathrm{Te}=100,000^{\circ} \mathrm{K}$.

(4) The DC star L 1363-3 which shows no features, has a temperature of approximately $10,000^{\circ} \mathrm{K}$.

(5) The DF and DG stars range from $4,500^{\circ} \mathrm{K}$ to $9,000^{\circ} \mathrm{K}$.

The temperatures tend to be higher than all previous estimates. For example, Weidemann (1963) found for $40 \mathrm{Eri} \mathrm{B} \mathrm{Te}=13,000^{\circ} \mathrm{K}$; Oke (1963) using Weidemann's models obtained $14,400^{\circ} \mathrm{K}$; Matsushima and Terashita (1969) obtained $15,300^{\circ} \mathrm{K}$ using their recent models; in this study these same models yield $\mathrm{Te}=16,500^{\circ} \mathrm{K}$, due largely to the change in the calibration of $\alpha$ Lyrae.

\section{References}

Eggen, O. J. , and Greenstein, J. L. 1965, Ap. J., 141, 83. Matsushima, S., and Terashita, Y. 1969, Ap.J. , $\overline{156}, 219$. Oke, J. B. 1963, "The Observations of White Dwarf Stars"

(Paper presented at the Cleveland meeting of the AAAS ).

Oke, J. B., and Schild, R.E. 1970, Ap. J. (in press). Terashita, Y. , and Matsushima, S. 1969, Ap. J., 156, 203. Weidemann, V. 1963, Zs. f. Ap., 57, 87. 


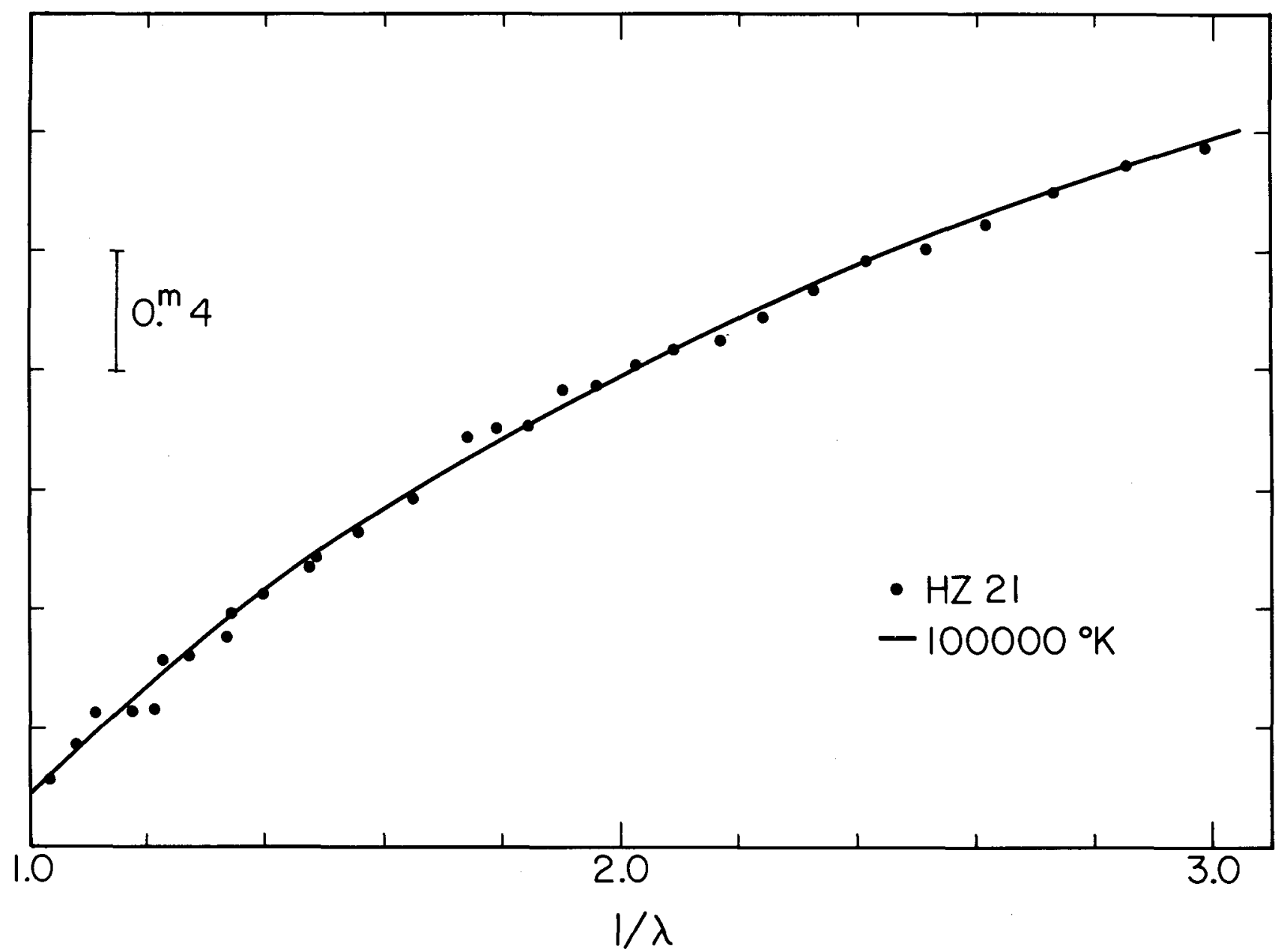

Figure 1. HZ 21. According to Eggen and Greenstein (1965), this star has $\mathrm{H}, \mathrm{He}$ I, and He II lines. It may be a subdwarf $\mathrm{O}$ star rather 


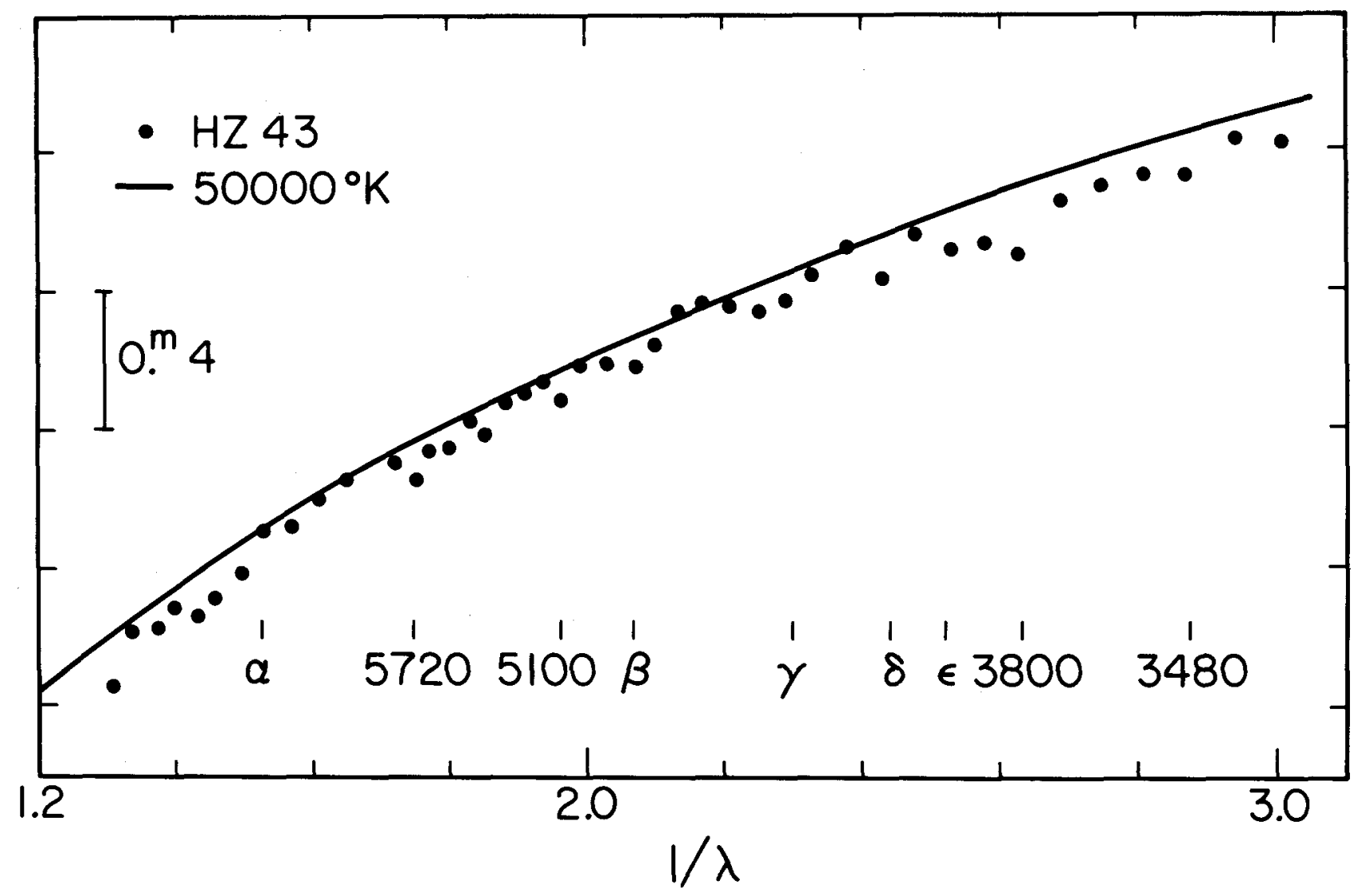

Figure 2. HZ 43. This star has a faint cool companion at a separation of about 3 ". This energy distribution may be slightly contaminated by this red star. The black-body temperature is $50,000^{\circ} \mathrm{K}$. Hydrogen lines are weak (Eggen and Greenstein 1965). 


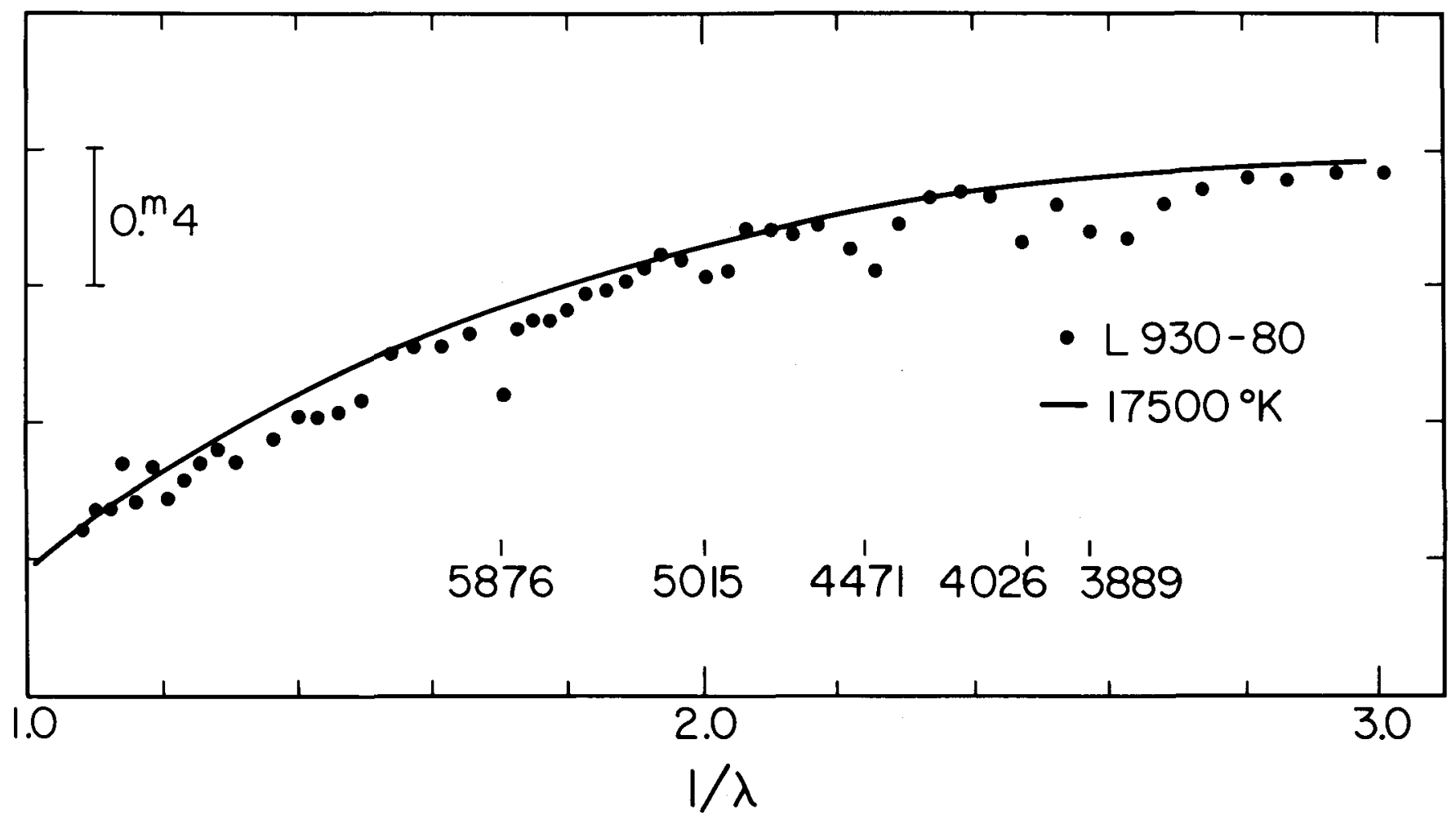

Figure 3. L 930-80. This is spectral type DB and has strong Helium lines. The black body temperature is $17,500^{\circ} \mathrm{K}$. 


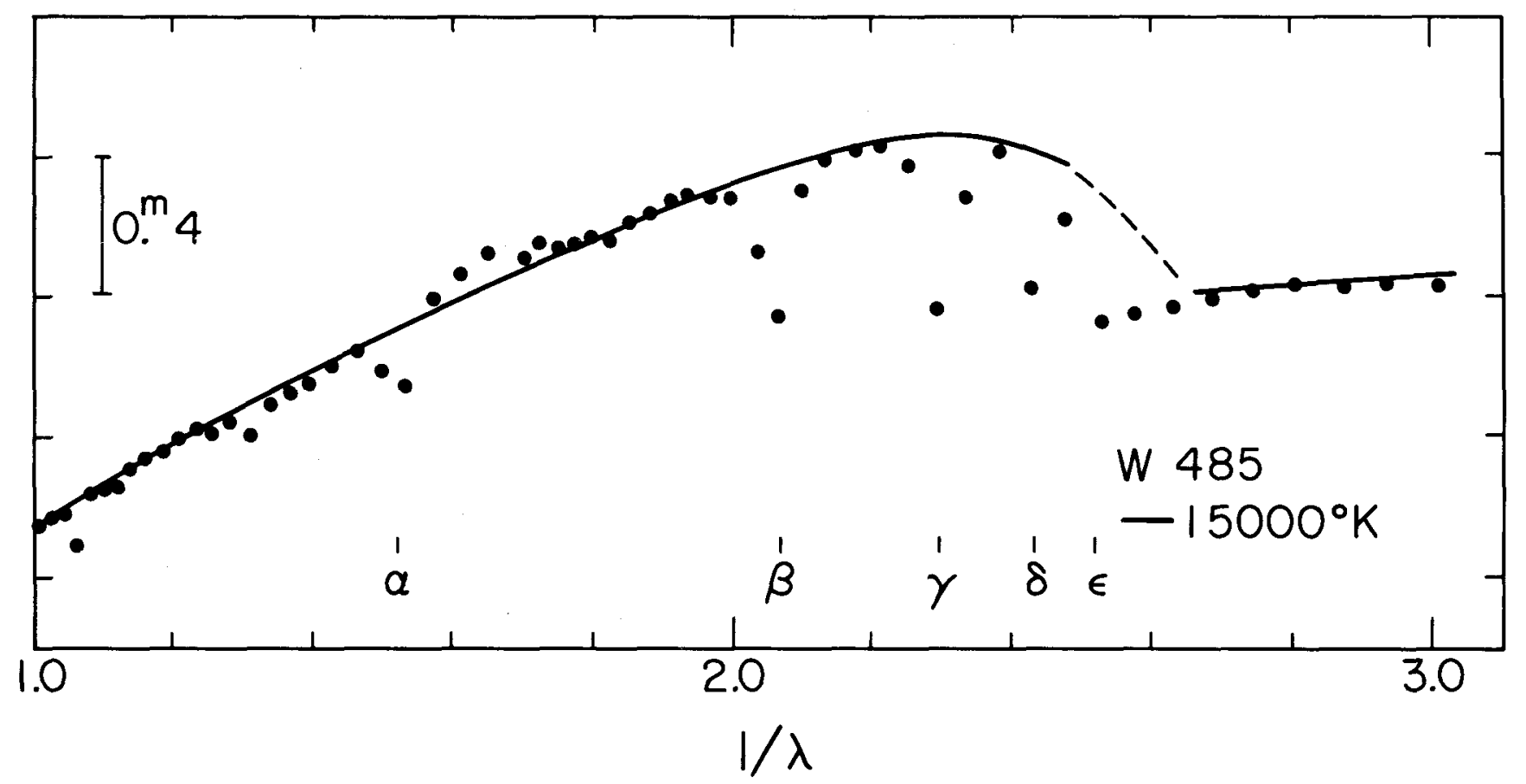

Figure 4. W485. This is a typical white dwarf of type DA. The fit to the model of Terashita and Matsushima (1969) is excellent. The absolute magnitude $M_{v}=10.8$ from the trigonometric parallax. 


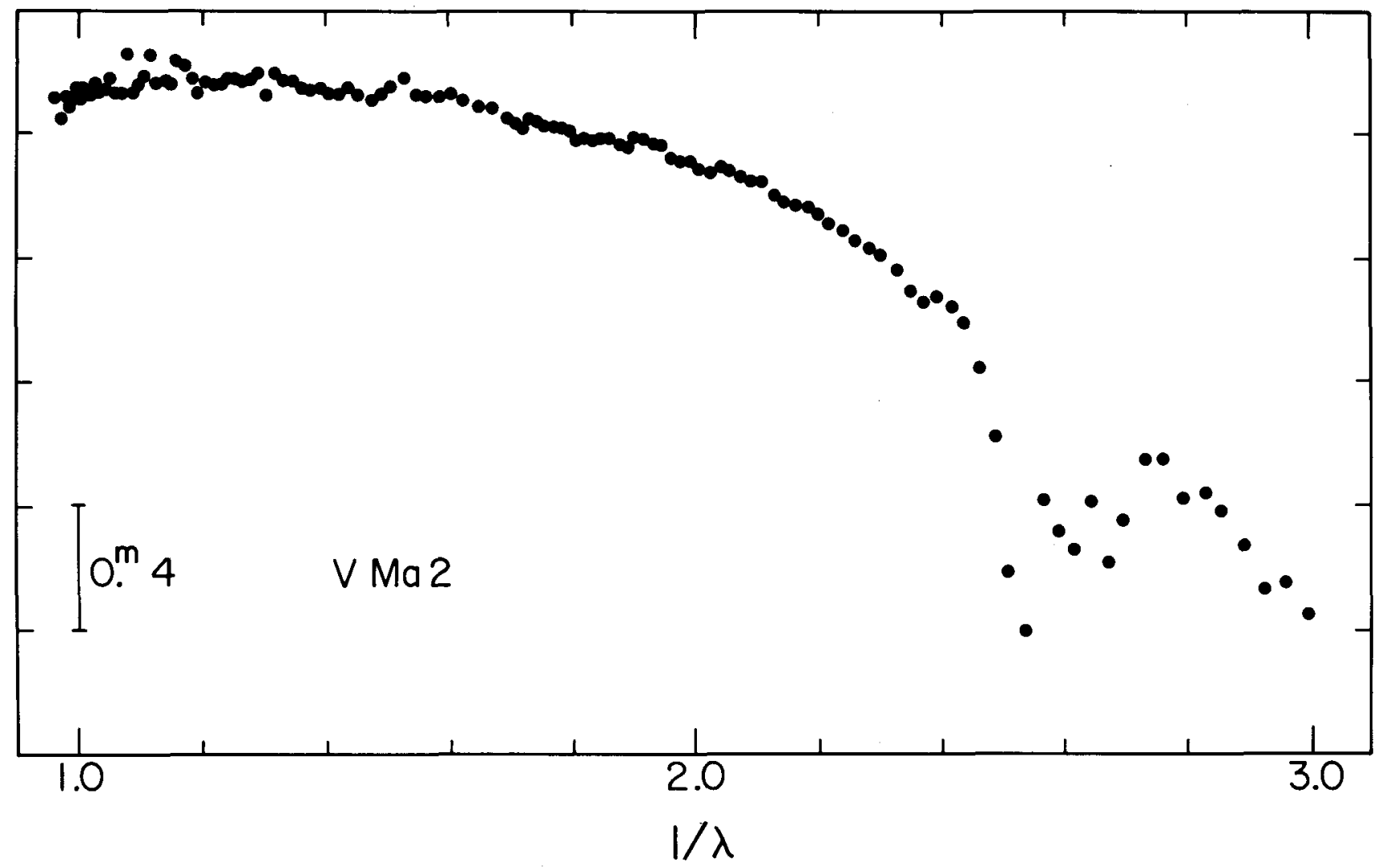

Figure 5. VMa 2. This is the best-known DG star. The effective temperature must be less than $5,000^{\circ} \mathrm{K}$; no models exist as yet. The star has strong metal lines in the ultraviolet, as well as $\mathrm{H}$ and $\mathrm{K}$ of $\mathrm{Ca}$ II (Eggen and Greenstein 1965). $\mathrm{M}_{\mathrm{v}}=14.2$ from 